\title{
Adaptation of a smart walker for stroke individuals: a study on sEMG and accelerometer signals
}

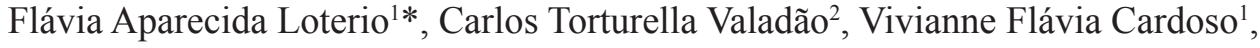 \\ Alexandre Pomer-Escher ${ }^{1}$, Teodiano Freire Bastos ${ }^{1,2}$, Anselmo Frizera-Neto ${ }^{2}$ \\ ${ }^{1}$ Postgraduate Program in Biotechnology, Federal University of Espirito Santo, Vitória, ES, Brazil. \\ ${ }^{2}$ Postgraduate Program in Electrical Engineer, Federal University of Espirito Santo, Vitória, ES, Brazil.
}

\begin{abstract}
Introduction: Stroke is a leading cause of neuromuscular system damages, and researchers have been studying and developing robotic devices to assist affected people. Depending on the damage extension, the gait of these people can be impaired, making devices, such as smart walkers, useful for rehabilitation. The goal of this work is to analyze changes in muscle patterns on the paretic limb during free and walker-assisted gaits in stroke individuals, through accelerometry and surface electromyography (sEMG). Methods: The analyzed muscles were vastus medialis, biceps femoris, tibialis anterior and gastrocnemius medialis. The volunteers walked three times on a straight path in free gait and, further, three times again, but now using the smart walker, to help them with the movements. Then, the data from gait pattern and muscle signals collected by sEMG and accelerometers were analyzed and statistical analyses were applied. Results: The accelerometry allowed gait phase identification (stance and swing), and sEMG provided information about muscle pattern variations, which were detected in vastus medialis (onset and offset; $p=0.022$ ) and biceps femoris (offset; $\mathrm{p}=0.025$ ). Additionally, comparisons between free and walker-assisted gaits showed significant reduction in speed (from 0.45 to $0.30 \mathrm{~m} / \mathrm{s} ; \mathrm{p}=0.021$ ) and longer stance phase (from 54.75 to $60.34 \%$; $\mathrm{p}=0.008$ ). Conclusions: Variations in muscle patterns were detected in vastus medialis and biceps femoris during the experiments, besides user speed reduction and longer stance phase when the walker-assisted gait is compared with the free gait.
\end{abstract}

Keywords Stroke, sEMG, Smart walker, Gait, Accelerometer.

\section{Introduction}

Stroke is considered a major health issue worldwide, since it is a leading cause of motor disabilities, affecting the independence and ability to perform daily tasks in most cases (Belda-Lois et al., 2011; World..., 2015). There are two distinct types of stroke: the ischemic and the hemorrhagic. The first one is the most common and is responsible for $85-90 \%$ of cases, while the second type occurs in a smaller number (10-15\%). In contrast, the mortality rate ranges from 8 to $12 \%$ for the ischemic

This is an Open Access article distributed under the terms of the Creative Commons Attribution License, which permits unrestricted use, distribution, and reproduction in any medium, provided the original work is properly cited.

How to cite this article: Loterio FA, Valadão CT, Cardoso VF, Pomer-Escher A, Bastos TF, Frizera-Neto A. Adaptation of a smart walker for stroke individuals: a study on sEMG and accelerometer signals. Res Biomed Eng. 2017; 33(4):293-300. DOI: 10.1590/24464740.01717

*Corresponding author: Postgraduate Program in Biotechnology, Federal University of Espirito Santo, Fernando Ferrari Ave, 514, Goiabeiras, CEP29075-910, Vitória, ES, Brazil. E-mail: loteriofa.ufes@gmail.com Received: 13 April 2017/ Accepted: 16 September 2017 type, while the hemorrhagic type has more fatal outcomes with numbers varying between $33 \%$ and $45 \%$ (Ovbiagele and Nguyen-Huynh, 2011).

Aside from the stroke type, the location and extension of the brain lesions may lead to different sequels (Deb et al., 2010) and, due to this reason there is a high heterogeneity among stroke sequels (Belda-Lois et al., 2011), varying according to the brain lesion location and extension. A lesion that occurs in the anterior cerebral artery, for example, may cause motor injuries predominantly in the lower extremity of the contralateral side, which interfere in the gait and body balance (Pare and Kahn, 2012).

Patients that had stroke usually have spastic muscles in the quadriceps femoris (vastus medialis, vastus lateralis, vastus intermedius and rectus femoris) and triceps surae (gastrocnemius medialis, gastrocnemius lateralis and soleus) while the hamstrings (biceps femoris, semitendinosus and semimembranosus) and tibialis anterior are flaccid, hindering the knee flexion and dorsiflexion (Murray et al., 2014; Sheffler and Chae, 2015). In spite of flexor weakness, stroke individuals present more co-contractions between agonist and 
antagonist muscles when compared with healthy subjects (Shao et al., 2009), which occur in order to avoid knee and plantar hyperflexion.

All these conditions create a tendency on stroke individuals to produce a compensatory movement in order to walk, which is known as hip circumduction, typical in stroke gait (Whittle, 2007), causing an asymmetric gait, and overloading the non-paretic limb.

Due to this asymmetry and lack of balance, about $75 \%$ of stroke patients need assistance for walking independently during the first three months after stroke onset (Verma et al., 2012). However, there are no evidence-based criteria for choosing the device to help the patient (Verma et al., 2012). Tyson and Rogerson (2009) evaluated the use of cane and foot-ankle orthosis, which provided confidence and safety to the patients (20 stroke patients; mean age: $65.6 \pm 10.4$ years; mean time since stroke: $6.5 \pm 5.7$ weeks), improving their functional mobility. On the other hand, Suica et al. (2016) analyzed the immediate effect using a rollator, although for healthy subjects (19 subjects; 22 to 70 years), identifying a reduced muscle activity of the lower limbs (gluteus medius and maximus, rectus femoris, semitendinosus, tibialis anterior and gastrocnemius) caused by the weight bearing imposed on the walker.

Most stroke individuals need rehabilitation, whose main goal is the movement recovery to allow them to carry out daily tasks independently (Dohring and Daly, 2008; Roger et al., 2011). This rehabilitation depends on many factors: lesion severity, age, type of therapeutic intervention, and how complex the stroke was. However, in many cases, rehabilitation does not provide an efficient recovery, and sometimes worsening the clinical status and the damage in the non-paretic limb. In such cases, those therapeutic interventions may provoke decreased mobility and secondary complications (Allen et al., 2011). On the other hand, conventional gait training and rehabilitation, commonly used nowadays, may not provide a total restoration for most patients (Dohring and Daly, 2008; Suica et al., 2016).

Many studies (Cifuentes et al., 2014; Dohring and Daly, 2008; Tan et al., 2013) used robotic devices for motor rehabilitation, to recover important features of the gait and maintain muscle integrity. However, to the extent of our knowledge, no neuromuscular analysis was performed using robotic walkers applied for stroke rehabilitation. The main goal of this paper is to analyze changes in the muscle pattern on paretic limb during free and walker-assisted gaits in stroke individuals, through accelerometry and surface electromyography (sEMG). Another important goal is to verify the volunteer adaptation to a smart walker in the first contact. Therefore, this study is focused on the pattern-variation analysis of the paretic limb muscles and the swing and stance phase duration, in addition to the walking speed during the use of robotic walker and in free gait.

\section{Methods}

\section{Volunteers}

Eight ischemic stroke individuals (4 males and 4 females; $65.75 \pm 6.27$ years old), from a rehabilitation institution of Espirito Santo state (Brazil), volunteered for the experiments. The number of volunteers generated a sample size for this study that has an effect size of 0.8 , with statistical power of $50 \%$ and alpha equals 0.05 . The research was previously approved by the Ethical Committee of Federal University of Espírito Santo (UFES/Brazil) and all volunteers signed the informed consent.

Eligibility criteria for inclusion in this study were: only one stroke that happened at least from 6 months up to 5 years before the tests; hemiparetic gait; Functional Ambulation Classification - FAC (Holden et al., 1984) in stage 2 or higher; ability to remain erect and with elbows at $90^{\circ}$ while using the smart walker; age range from 50 to 80 years; enough cognitive skills and language to follow the experiment instructions. Individuals were excluded if they could not walk independently, had any musculoskeletal or neurological disorder limiting ambulation unrelated to the stroke, and if they had cardiorespiratory impairment, conditions that may prevent them from performing walking tests. Each volunteer was classified through a functional walking test (FAC) by the same physiotherapist, who has more than 20 years of experience.

\section{sEMG and accelerometer data}

All procedures for sEMG data acquisition and processing were based on recommendations of the "Standards for reporting EMG data" (Merletti and Torino, 2015). The kind of electrodes used was $\mathrm{Ag} / \mathrm{AgCl}$ discoid shape, with $10 \mathrm{~mm}$ diameter, pre-gelled and with inter-electrode distance of $20 \mathrm{~mm}$. Before the electrode placement, the skin was cleaned (alcohol 70\%) and shaved to reduce impedance. Signals from four muscles of lower limb - vastus medialis (VM), biceps femoris (BF), tibialis anterior (TA) and gastrocnemius medialis (GM) - were acquired and analyzed. In addition, a reference electrode was placed on the medial malleolus. In all cases, the analyzed limb was the contralateral to the brain lesion. For better accuracy in electrode placement, two experts checked the electrode position placed on the muscles. Cables from the sEMG acquisition equipment were fixed on the limb using adhesive tape to minimize motion artifacts. In addition, a biaxial accelerometer was fixed using adhesive tape on the ankle of the 
contralateral limb, with the y-axis pointing cranially and $\mathrm{x}$-axis pointing anteriorly.

Both sEMG and accelerometer data were recorded simultaneously using an acquisition equipment EMG 830C (EMG System do Brasil Ltda ${ }^{\circledR}$ ) with 16-bit analog/digital conversion resolution, amplifier gain up to $2000 \mathrm{~V} / \mathrm{V}$, common mode rejection $>100 \mathrm{~dB}$, input impedance of $10^{9} \Omega$, and maximum sampling frequency of $2 \mathrm{kHz}$. The measurement capacity ranged from -2000 to $2000 \mu \mathrm{V}$ with sensitivity of $0.061 \mu \mathrm{V}$.

\section{Smart walker}

A smart walker from UFES/Brazil (Valadão et al., 2016) (Figure 1) was used in the experiments, which was built from a conventional four-legged walker adapted to a robotic mobile platform. The smart walker structure has forearm bars to provide weight support and comfort during its use, also allowing the user to guide it. The smart walker has also a height adjustment, which allows the user to stay in an upright posture. An onboard laser sensor is used to provide information about the distance from the walker to the user's leg. By using the information provided by the laser sensor, the walker can adjust its speed through a proportional-integral-derivative controller (PID), with the goal of keeping the user at a predefined distance and angle, thus aiding him/her to maintain right posture (position and orientation) while using the device.

In the experiments, the walker slows down when the user is within a distance greater than the set-point, allowing the user to get closer to the walker. In the opposite case, i.e., if the user is closer than the set-point, the walker moves away to make him/her catch the set-point. In general, the walker makes the user stays always on the central angle and at the predefined set-point posture (distance: $70 \mathrm{~cm}$; orientation: $0^{\circ}$ ) (Valadão et al., 2016).
To enhance the experience with the walker, all tests were carried out using a classical PID controller together with a safety supervisor system composed of a set of rules (Table 1) to avoid harmful situations for the user while using the walker (Valadão et al., 2016).

\section{Experimental protocol}

The experimental protocol was performed in two stages. Firstly, the volunteer walked freely for 10 meters in a straight path on flat ground at a comfortable speed (chosen by the volunteer). This walking was performed three times and there was one minute of resting time to avoid fatigue. Secondly, there was a time for adaptation and instruction regarding the use of the smart walker, which varied according to the needs of each volunteer. Following, the volunteer performed the same path three times, but now with the assistance of the smart walker.

\section{Data analysis}

Once collected to the computer, the signals were analyzed to identify the gait phases and muscle activity. From the accelerometer signals, the analysis was done following the method of Han et al. (2009), in which the gait cycle begins with the heel strike and ends in the next heel strike of the same foot, corresponding to $100 \%$ of the gait cycle.

Table 1. Security rules of the smart walker.

\begin{tabular}{cc}
\hline Situation & Action \\
\hline Do not detect lower limbs & Stop immediately \\
Detect only one lower limb & Stop immediately \\
Negative linear speed (robot) & Stop immediately \\
Speed above allowed & Limit to maximum allowed \\
Lower limbs too close & Increase the speed to move \\
& away from the user \\
Lower limbs too far & Reduce slowly until stop \\
\hline
\end{tabular}
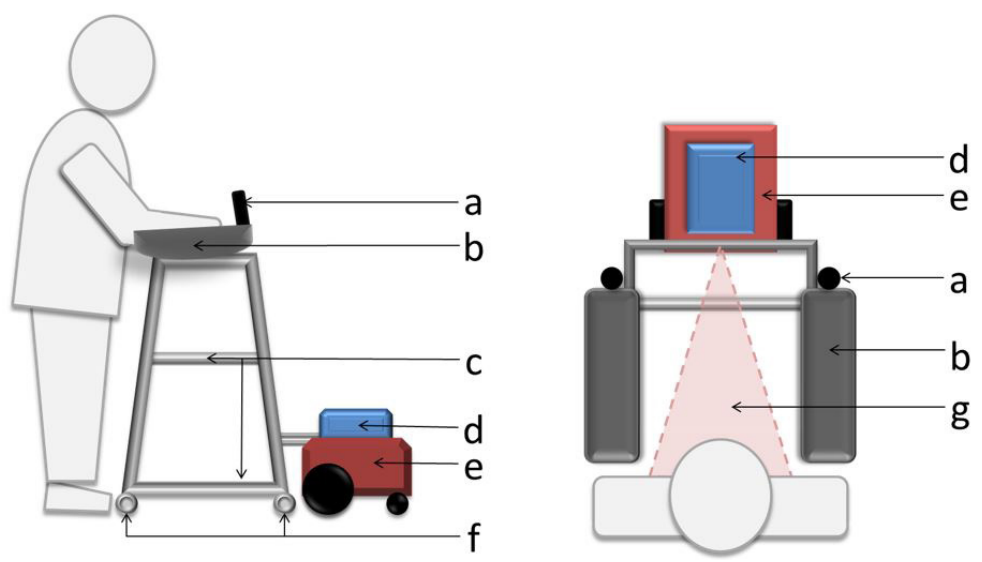

Figure 1. Smart Walker scheme: side view (left) and top view (middle). Stroke subject using the walker (right) in an experiment. Structure changes in the walker: (a) Handlebar; (b) Forearm support; (c) Stabilizer bars; (d) Laser sensor; (e) Pioneer 3-DX robot; (f) Free wheels; (g) Fixed distance $(70 \mathrm{~cm})$ from the user to laser sensor. 
The sEMG raw signals were full-wave rectified and filtered using a $10-500 \mathrm{~Hz}$ band-pass filter to minimize the influence of motion artefacts and for signal smoothing. In addition, a normalization was applied using the method of finding the maximum peak during the movement, which is considered the best method for dynamics contractions analyses and for neurologic patients as they are not able to produce a reliable maximum contraction (Perry and Burnfield, 2010).

At least 20 cycles of each volunteer were acquired and the average onset and offset were found calculating the RMS (root mean square) of the signal, indicating the mean pattern for each muscle. The results were presented as mean and standard deviation (SD), followed by coefficient of variation (CV), which is calculated as $(\mathrm{SD} /$ mean) $\mathrm{x} 100$. Samples with $\mathrm{CV}$ values lower than $15 \%$ are considered to have low dispersion; from 15 to $30 \%$ moderate dispersion; and, higher than $30 \%$, high dispersion.

For statistical analysis, using the Shapiro-Wilk normality test, we identified that the samples are not normally distributed. Therefore, the Wilcoxon signed-rank test was applied to compare the free and walker-assisted gaits and to verify if there was a significant difference between them. When p-value $<0.05$, the null hypothesis was rejected, which considers there is no difference between the free and walker-assisted gaits.

\section{Results}

Eight volunteers that had suffered stroke participated of the experiments (with the height of the smart walker forearm support adjusted for each one) and their physical information is arranged on Table 2. Although there had been a great elapse of time after stroke $(2.83 \pm 1.51$ years, CV: $53 \%$ ), all of volunteers showed typical stroke gait, in which they had knee hyperextension and plantar hyperflexion and, consequently, hip circumduction.

\section{Speed}

During the free gait, the volunteers were instructed to walk at a comfortable speed. The average speed for the three repetitions (Table 3 ) was recorded and the mean of the eight volunteers was $0.45 \pm 0.06 \mathrm{~m} / \mathrm{s}$. The smart walker automatically adjusts its speed according to the user's speed, but it is possible to change its speed in any moment, according to therapist's specification. Due to safety concerns, the smart walker maximum speed was limited at $0.50 \mathrm{~m} / \mathrm{s}$. The average speed reached by the patients during the walker-assisted gait was $0.30 \pm 0.07 \mathrm{~m} / \mathrm{s}$, which represents a reduction of $33.3 \%$ compared with the free gait. There was statistically significant difference between these gaits $(p=0.021)$, which means there was a decrease in the speed when the patients used the walker as assistance for walking.

\section{Accelerometer and cycle phases}

Some researchers (Han et al., 2009; Lee et al., 2010; Saremi et al., 2006) have used accelerometer signals to divide the gait in stance and swing phases by finding specific peaks (Figure 2), in which toe-off indicates the end of the stance phase and, consequently, the beginning of the swing phase. The average percentage of toe-off during the free gait was $54.75 \% \pm 7.04$, and during the assisted gait it was $60.34 \% \pm 8.10$ (Table 4). Using the Wilcoxon's test ( $\alpha=0.05$ ), the $p$-value was 0.008 . Therefore, there was a statistically significant difference, and an increase in the stance phase using the smart walker could be observed.

\section{sEMG and muscle pattern}

After dividing the gait phases, the muscle activation patterns for each individual were determined (Figure 2). $\mathrm{VM}$ and $\mathrm{BF}$ muscles presented longer activation period. For volunteers with higher GM spasticity (V1, V2, V4 and V5), the TA muscle had longer activation period, due to coactivation to control the plantar flexion. All the volunteers showed an intense activation of GM

Table 2. Volunteers' physic information.

\begin{tabular}{|c|c|c|c|c|c|c|c|c|c|}
\hline Volunteer & 1 & 2 & 3 & 4 & 5 & 6 & 7 & 8 & Mean \\
\hline Gender & M & M & M & $\mathrm{F}$ & $\mathrm{F}$ & M & $\mathrm{F}$ & $\mathrm{F}$ & $4 \mathrm{M} / 4 \mathrm{~F}$ \\
\hline Height (cm) & 168 & 160 & 176 & 160 & 165 & 175 & 162 & 163 & $166.12 \pm 6.36$ \\
\hline Age (years) & 77 & 60 & 63 & 62 & 65 & 61 & 74 & 54 & $64.50 \pm 7.54$ \\
\hline More affected side & $\mathrm{R}$ & $\mathrm{L}$ & $\mathrm{L}$ & $\mathrm{R}$ & $\mathrm{R}$ & $\mathrm{R}$ & $\mathrm{L}$ & $\mathrm{L}$ & $4 \mathrm{R} / 4 \mathrm{~L}$ \\
\hline Time after stroke (years) & 0.9 & 0.6 & 4.3 & 5.0 & 3.0 & 2.5 & 3.2 & 3.1 & $2.83 \pm 1.51$ \\
\hline FAC & 4 & 3 & 2 & 3 & 4 & 3 & 4 & 5 & $3,50 \pm 0.93$ \\
\hline
\end{tabular}

M: male; F: female; R: right; L: left; FAC is the acronym for Functional Ambulation Category.

Table 3. Average speed (m/s) during free and walker-assisted gait.

\begin{tabular}{cccccccccccc}
\hline Volunteer & $\mathbf{1}$ & $\mathbf{2}$ & $\mathbf{3}$ & $\mathbf{4}$ & $\mathbf{5}$ & $\mathbf{6}$ & $\mathbf{7}$ & $\mathbf{8}$ & Mean & SD & p-value \\
\hline Free gait & 0.47 & 0.40 & 0.43 & 0.46 & 0.40 & 0.42 & 0.46 & 0.59 & 0.45 & 0.06 & $0.021^{*}$ \\
Assisted gait & 0.32 & 0.44 & 0.27 & 0.34 & 0.28 & 0.27 & 0.24 & 0.23 & 0.30 & 0.07 & \\
\hline
\end{tabular}

\footnotetext{
* Statistically significant with p-value $<0.05-$ Wilcoxon's test.
} 

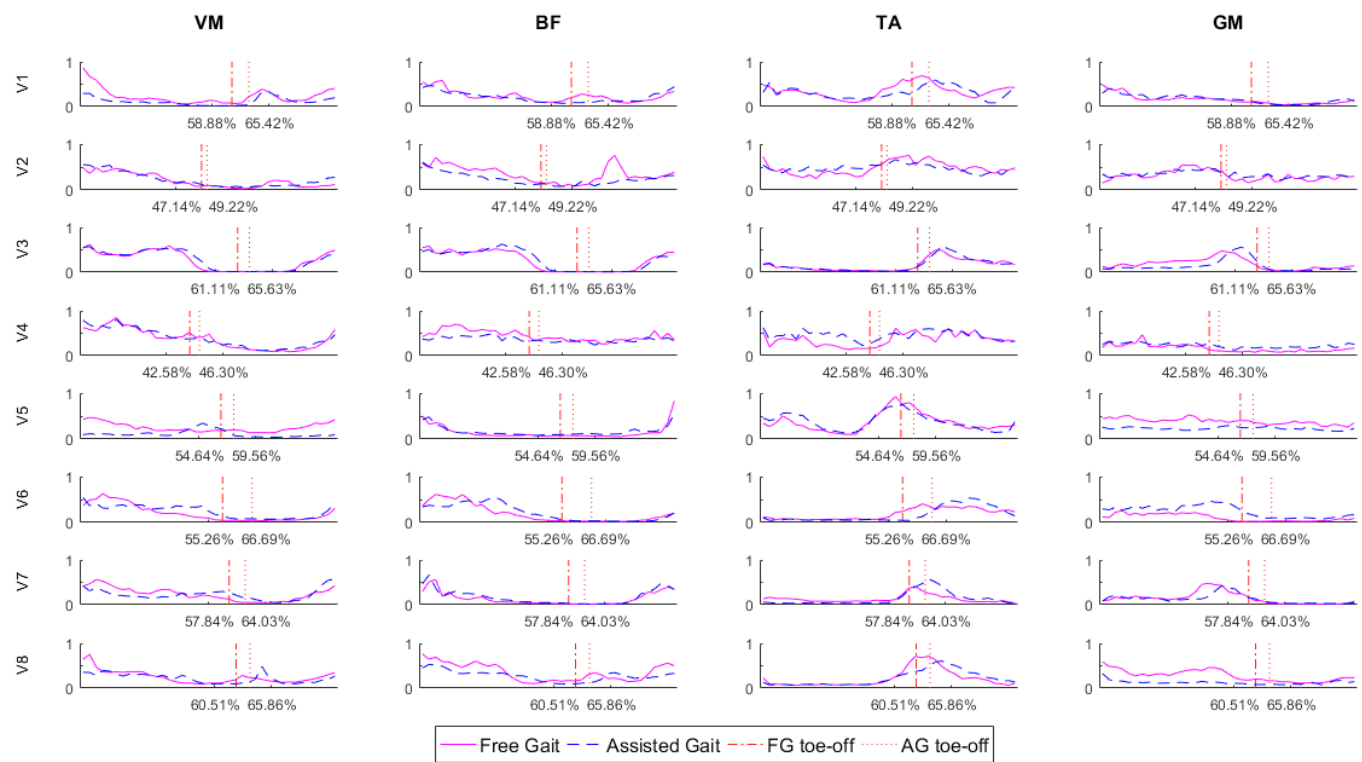

Figure 2. Average muscle pattern (EMG signals) for each individual during free (continuous pink curve) and walker-assisted gait (dotted blue curve). The red lines represent the toe-off, which are dividing the cycle in stance, and swing phase. VM (vastus medialis); BF (biceps femoris); TA (tibialis anterior); GM (gastrocnemius medialis); FG (free gait); AG (assisted gait); V1 (first volunteer); V2 (second volunteer) and so on.

Table 4. Comparison of duration of stance phase (toe-off) and muscle onset/offset between free and walker-assisted gait.

\begin{tabular}{|c|c|c|c|c|c|c|c|c|}
\hline & & \multicolumn{3}{|c|}{ Free Gait } & \multicolumn{3}{|c|}{ Assisted Gait } & \multirow{2}{*}{ p-value } \\
\hline & & Mean (\%) & SD & CV (\%) & Mean (\%) & SD & CV (\%) & \\
\hline \multicolumn{2}{|c|}{ Toe-off } & 54.75 & 6.62 & 12.09 & 60.34 & 8.10 & 13.42 & $0.008 *$ \\
\hline \multirow[t]{2}{*}{ VM } & on & 77.56 & 7.72 & 9.95 & 83.58 & 7.79 & 9.32 & $0.022 *$ \\
\hline & off & 50.32 & 8.61 & 17.11 & 56.41 & 9.09 & 16.11 & $0.022 *$ \\
\hline \multirow[t]{2}{*}{ BF } & on & 78.20 & 9.49 & 12.14 & 80.32 & 6.61 & 8.23 & 0.236 \\
\hline & off & 41.67 & 6.54 & 15.69 & 48.08 & 7.72 & 16.06 & $0.025^{*}$ \\
\hline \multirow[t]{2}{*}{ TA } & on & 44.87 & 5.48 & 12.21 & 47.12 & 7.56 & 16.04 & 0.203 \\
\hline & off & 23.40 & 7.98 & 34.10 & 25.64 & 6.28 & 24.49 & 0.309 \\
\hline \multirow[t]{2}{*}{ GM } & on & 89.96 & 5.39 & 5.99 & 90.07 & 5.47 & 6.07 & 0.439 \\
\hline & off & 61.86 & 8.33 & 13.47 & 65.06 & 6.20 & 9.53 & 0.248 \\
\hline
\end{tabular}

* Statistically significant with p-value $<0.05$ - Wilcoxon's test. SD and CV mean standard deviation and coefficient of variation, respectively. VM and $\mathrm{BF}$ are the acronyms for the vastus medialis muscle and for the biceps femoris muscle, respectively. Additionally, the TA and GM acronyms refers to the tibialis anterior and gastrocnemius medialis muscles. Toe-off is the moment preceding the swing phase, when the toe loses the contact with the ground to start the swing phase of its leg.

muscle, with its onset occurring during the swing phase, a behavior not found in healthy gait.

The mean onset and offset were calculated for the muscles vastus medialis, biceps femoris, tibialis anterior and gastrocnemius medialis and the results are shown in Table 4. The CV (coefficient variation) value indicates how diversified the sample is. The VM/BF muscle offset and TA onset (only assisted gait) values presented medium dispersion (15-30\%), while the TA offset showed high dispersion (> 30\%). Contrastingly, all other values had low dispersion.

Comparing free and walker-assisted gait, there was statistically significant difference only in VM onset/offset $(p=0.022)$ and BF offset $(p=0.025)$, with highest percentage rate in the gait cycle. However, even with these values, the onset/offset remained in the same phase in both gaits.

\section{Discussion}

The development of robotic devices for rehabilitation should consider patient's residual abilities, activities they have to develop in their daily tasks and work, the device comfort and practical aspects (Helal et al., 2008). The employment of robotic devices for stroke rehabilitation has been widely studied as a new and efficient way to recover motor skills (Cifuentes et al., 2014; Dohring and Daly, 2008; Tan et al., 2013). However, it should be evaluated if there are changes during the assisted gait, such as asymmetry reduction in some gait parameters and muscle activation more similar to the normal activation. 
These data contribute to verify the possibility of using these robotic devices in rehabilitation clinics.

\section{Speed}

Compensatory movements and lower limb asymmetry cause changes in the gait patterns of stroke patients (Beaman et al., 2010), and the spasticity increases the oxygen demand (Lee et al., 2010; Roger et al., 2011). Moreover, age is another important factor as our sample is composed of elderly people (64.50 \pm 7.54 years). All these features cause a reduction in the gait speed.

Stroke gait speed varies among different levels of motor damages and stroke time. Thus, according to the literature, hemiparetic gait has a wide range from 0.10 to $1.00 \mathrm{~m} / \mathrm{s}$ (Balaban and Tok, 2014; Lamontagne et al., 2000). In this study, the speed was more homogeneous $(0.45 \pm 0.06 \mathrm{~m} / \mathrm{s})$, probably because the volunteers were in chronic phase (time after stroke $>6$ months).

In the experiments carried out with the smart walker the speed limit was of $0.5 \mathrm{~m} / \mathrm{s}$ (limited by the walker). In such cases, the highest speed achieved by volunteers was $0.44 \mathrm{~m} / \mathrm{s}$ and the average speed was $0.30 \pm 0.07 \mathrm{~m} / \mathrm{s}$ (statistically significance $\mathrm{p}=0.021$ ). Therefore, the speed limit was not the responsible for the user speed reduction. According to the literature (Martins et al., 2012), during the walker-assisted gait reduced speeds are already expected.

Furthermore, our smart walker is intended for gait rehabilitation, thus the gait speed is not necessarily related to the gait pattern improvement, and in case of gait training, the goal for the patients is to help them performing the task correctly and to stop using compensatory movements acquired after stroke. Better stability and homogeneous distribution of body weight on the lower limbs provides a gait closer to the natural one, with better symmetry between the lower limbs, including increasing the support phase of the paretic limb. In fact, a study (Dragin et al., 2014) of 4-week clinical trial (22 subacute stroke patients) using a body postural support connected to a powered rolling walker concluded that a smart walker changes the gait speed and balance control significantly when compared to a control group.

\section{Accelerometer and cycle phases}

To our knowledge, Saremi et al. (2006) was the only study that used an accelerometer to obtain kinematic parameters in stroke gait, finding the same pattern of peaks of healthy gait.

In our study, asymmetry between contralateral and ipsilateral limbs was observed in decreased duration of support on the contralateral limb (Allen et al., 2011; Dobrovolny et al., 2003); consequently, there is a difference between the step length of one limb and the other (Chen et al., 2005).

In studies of Lamontagne et al. (2000) with 30 subjects ( $<6$ months after stroke; mean age of 62.1 years; gait speed: $0.48 \mathrm{~m} / \mathrm{s}$ ), the toe-off occurred in $67 \%$ of the gait cycle. Another work (Den Otter et al., 2007) analyzed the gait of 24 individuals (mean of 8.75 months after stroke; mean age of 58.6 years; gait speed: $0.35 \mathrm{~m} / \mathrm{s}$ ), detecting the end of stance phase at $68 \%$ of the gait cycle. Comparing those data with these found in our study, the duration of the stance phase during the free gait was shorter: $54.75 \% \pm 7.04$.

Even with a slower speed, during the assisted gait, the toe-off phase was $60.34 \% \pm 8.10$ of the gait cycle, which represents a significant increase $(p=0.008)$. Due to the body stability needed for walking, the ipsilateral limb is overloaded in free gait; therefore, there is a higher support phase by the ipsilateral limb.

These data along with other earlier studies demonstrated that accelerometer can be helpful for gait analysis, as it allowed identifying the main cycle phases.

\section{sEMG and muscle pattern}

Spasticity and weakness are the main features of motor damages in stroke patients. Even due to the heterogeneity of stroke individuals, there are some common general characteristics in gait, such as premature onset, and prolonged duration of firing and peaks of activity that differ from normal gait (Balaban and Tok, 2014).

Due to weakness in BF, there is lower propulsion performed by the paretic limb (Routson et al., 2013). However, in order to avoid knee hyperextension, BF shows a higher activation time. In fact, a study led by Corrêa et al. (2005) indicated a coactivation between quadriceps and hamstrings muscle groups. Similarly, in our results, VM and BF showed a longer activation time than healthy gait, as expected during both free and walker-assisted gait.

Coactivation of TA/GM is generally not observed in healthy gait (Den Otter et al., 2007; Perry and Burnfield, 2010). Differently, in our study, TA values (onset and offset) showed a high dispersion, mainly because of differences in the sample. The volunteers 1,2, 4 and 5 had longer periods of activation and more spasticity in GM (identifiable by higher basal level of sEMG) than the others. We identified TA/GM coactivation in all subjects, mainly due to longer duration of the TA activation. Our results agree with studies of Shao et al. (2009), in which GM was active during the initial contact, in a moment when the plantar flexors are usually not active. We also observed GM activation beginning in the swing phase. This premature activation of GM can contribute to abnormal pressure distribution (Corrêa et al., 2005). On the other hand, a reduction of the excessive 
coactivation can decrease the higher energy cost associated with locomotion after stroke (Lamontagne et al., 2000).

To summarize, the major contribution of this research is the neuromuscular analysis in stroke individuals during assisted gait by robotic walker and the main feature found was a longer stance phase in the walker-assisted gait compared to the free gait. In addition, the results of this study regarding muscle activation during assisted gait showed statistically significant differences in $\mathrm{VM}$ and $\mathrm{BF}$ offset, and VM onset, when compared to the free walking. Tibialis anterior (TA) and gastrocnemius medialis (GM) showed more time of coactivation in four out of eight volunteers.

Another important contribution found in this work was the opinion reported by stroke individuals, after their first contact with the robotic walker. These individuals reported they could easily get adapted to the robotic walker and this one was useful and helpful. In future studies, clinical analysis will be conducted for longer time regarding the use of the smart walker during rehabilitation for acute stroke patients.

\section{Acknowledgements}

The contents of this publication were developed under a grant from CNPq, CAPES and FAPES (Brazil). The authors have no conflicts of interest to declare.

\section{References}

Allen JL, Kautz SA, Neptune RR. Step length asymmetry is representative of compensatory mechanisms used in poststroke hemiparetic walking. Gait Posture. 2011; 33(4):538-43. PMid:21316240. http://dx.doi.org/10.1016/j.gaitpost.2011.01.004.

Balaban B, Tok F. Gait disturbances in patients with stroke. PM R. 2014; 6(7):635-42. PMid:24451335. http://dx.doi. org/10.1016/j.pmrj.2013.12.017.

Beaman CB, Peterson CL, Neptune RR, Kautz SA. Differences in self-selected and fastest-comfortable walking in poststroke hemiparetic persons. Gait Posture. 2010; 31(3):311-6. PMid:20006505. http://dx.doi.org/10.1016/j.gaitpost.2009.11.011.

Belda-Lois JM, Mena-del Horno S, Bermejo-Bosch I, Moreno JC, Pons JL, Farina D, Iosa M, Molinari M, Tamburella F, Ramos A, Caria A, Solis-Escalante T, Brunner C, Rea M. Rehabilitation of gait after stroke: a review towards a top-down approach. J Neuroeng Rehabil. 2011; 8(1):66. PMid:22165907. http://dx.doi.org/10.1186/1743-0003-8-66.

Chen G, Patten C, Kothari DH, Zajac FE. Gait differences between individuals with post-stroke hemiparesis and nondisabled controls at matched speeds. Gait Posture. 2005; 22(1):51-6. PMid:15996592. http://dx.doi.org/10.1016/j. gaitpost.2004.06.009.

Cifuentes CA, Rodriguez C, Frizera-Neto A, Bastos-Filho TF, Carelli R. Multimodal human-robot interaction for walkerassisted gait. IEEE Syst J. 2014; 10(3):933-43. http://dx.doi. org/10.1109/JSYST.2014.2318698.
Corrêa FI, Soares F, Andrade DV, Gondo RM, Peres JA, Fernandes AO, Corrêa JC. Muscle activity during gait following stroke. Arq Neuropsiquiatr. 2005; 63(3B):847-51. PMid:16258668.

Deb P, Sharma S, Hassan KM. Pathophysiologic mechanisms of acute ischemic stroke: An overview with emphasis on therapeutic significance beyond thrombolysis. Pathophysiology. 2010; 17(3):197-218. PMid:20074922. http://dx.doi.org/10.1016/j. pathophys.2009.12.001.

Den Otter AR, Geurts ACH, Mulder T, Duysens J. Abnormalities in the temporal patterning of lower extremity muscle activity in hemiparetic gait. Gait Posture. 2007; 25(3):342-52. PMid:16750632. http://dx.doi.org/10.1016/j.gaitpost.2006.04.007.

Dobrovolny CL, Ivey FM, Rogers MA, Sorkin JD, Macko RF. Reliability of treadmill exercise testing in older patients with chronic hemiparetic stroke. Arch Phys Med Rehabil. 2003; 84(9):1308-12. PMid:13680566. http://dx.doi.org/10.1016/ S0003-9993(03)00150-3.

Dohring ME, Daly JJ. Automatic synchronization of functional electrical stimulation and robotic assisted treadmill training. IEEE Trans Neural Syst Rehabil Eng. 2008; 16(3):310-3. PMid:18586610. http://dx.doi.org/10.1109/TNSRE.2008.920081.

Dragin A, Konstantinović L, Veg A, Schwirtlich L. Gait training of poststroke patients assisted by the Walkaround (body postural support). Int J Rehabil Res. 2014; 37(1):22-8. PMid:23820295. http://dx.doi.org/10.1097/MRR.0b013e328363ba30.

Han J, Jeon HS, Yi WJ, Jeon BS, Park KS. Adaptive windowing for gait phase discrimination in Parkinsonian gait using 3-axis acceleration signals. Med Biol Eng Comput. 2009; 47(11):1155-64. PMid:19693612. http://dx.doi.org/10.1007/ s11517-009-0521-5.

Helal AS, Mokhtari M, Abdulrazak B. The engineering handbook of smart technology for aging, disability, and independence. New Jersey: John Wiley \& Sons; 2008.

Holden MK, Gill KM, Magliozzi MR, Nathan J, Piehl-Baker L. Clinical gait assessment in the neurologically impaired. Reliability and meaningfulness. Phys Ther. 1984; 64(1):35-40. PMid:6691052. http://dx.doi.org/10.1093/ptj/64.1.35.

Lamontagne A, Richards CL, Malouin F. Coactivation during gait as an adaptive behavior after stroke. J Electromyogr Kinesiol. 2000; 10(6):407-15. PMid:11102843. http://dx.doi. org/10.1016/S1050-6411(00)00028-6.

Lee JA, Cho SH, Lee YJ, Yang HK, Lee JW. Portable activity monitoring system for temporal parameters of gait cycles. J Med Syst. 2010; 34(5):959-66. PMid:20703612. http://dx.doi. org/10.1007/s10916-009-9311-8.

Martins MM, Santos CP, Frizera-Neto A, Ceres R. Assistive mobility devices focusing on Smart Walkers: classification and review. Robot Auton Syst. 2012; 60(4):548-62. http://dx.doi. org/10.1016/j.robot.2011.11.015.

Merletti AR, Torino P. Standards for reporting EMG data. J Electromyogr Kinesiol. 2015; 24(2):1-2.

Murray M, Hardee A, Goldberg RL, Lewek MD. Loading and knee flexion after stroke: Less does not equal more. J Electromyogr Kinesiol. 2014; 24(1):172-7. PMid:24210795. http://dx.doi.org/10.1016/j.jelekin.2013.10.006. 
Ovbiagele B, Nguyen-Huynh MN. Stroke epidemiology: advancing our understanding of disease mechanism and therapy. Neurotherapeutics. 2011; 8(3):319-29. PMid:21691873. http:// dx.doi.org/10.1007/s13311-011-0053-1.

Pare JR, Kahn JH. Basic Neuroanatomy and Stroke Syndromes. Emerg Med Clin North Am. 2012; 30(3):601-15. PMid:22974640. http://dx.doi.org/10.1016/j.emc.2012.05.004.

Perry J, Burnfield JM. Gait analysis: normal and pathological function. J Sports Sci Med. 2010; 9(2):353.

Roger VL, Go AS, Lloyd-Jones DM, Adams RJ, Berry JD, Brown TM, Carnethon MR, Dai S, de Simone G, Ford ES, Fox CS, Fullerton HJ, Gillespie C, Greenlund KJ, Hailpern SM, Heit JA, Ho PM, Howard VJ, Kissela BM, Kittner SJ, Lackland DT, Lichtman JH, Lisabeth LD, Makuc DM, Marcus GM, Marelli A, Matchar DB, McDermott MM, Meigs JB, Moy CS, Mozaffarian D, Mussolino ME, Nichol G, Paynter NP, Rosamond WD, Sorlie PD, Stafford RS, Turan TN, Turner MB, Wong ND, Wylie-Rosett J, Roger VL, Turner MB. Heart disease and stroke statistics - 2011 update. Circulation. 2011; 123(4):e18-209. PMid:21160056. http://dx.doi.org/10.1161/ CIR.0b013e3182009701.

Routson RL, Clark DJ, Bowden MG, Kautz SA, Neptune RR. The influence of locomotor rehabilitation on module quality and post-stroke hemiparetic walking performance. Gait Posture. 2013; 38(3):511-7. PMid:23489952. http://dx.doi.org/10.1016/j. gaitpost.2013.01.020.

Saremi K, Marehbian J, Yan X, Regnaux J, Elashoff R, Bussel B, Dobkin BH. Reliability and validity of bilateral thigh and foot accelerometry measures of walking in healthy and hemiparetic subjects. Neurorehabil Neural Repair. 2006; 20(2):297-305. PMid:16679506. http://dx.doi.org/10.1177/1545968306287171.

Shao Q, Bassett DN, Manal K, Buchanan TS. An EMG-driven model to estimate muscle forces and joint moments in stroke patients. Comput Biol Med. 2009; 39(12):1083-8. PMid:19818436. http://dx.doi.org/10.1016/j.compbiomed.2009.09.002.

Sheffler LR, Chae J. Hemiparetic Gait. Phys Med Rehabil Clin N Am. 2015; 26(4):611-23. PMid:26522901. http://dx.doi. org/10.1016/j.pmr.2015.06.006.

Suica Z, Romkes J, Tal A, Maguire C. Walking with a four wheeled walker (rollator) significantly reduces EMG lowerlimb muscle activity in healthy subjects. J Bodyw Mov Ther. 2016; 20(1):65-73. PMid:26891639. http://dx.doi.org/10.1016/j. jbmt.2015.06.002.

Tan R, Wang S, Jiang Y, Ishida K, Fujie MG. Path tracking control of an omni-directional walker considering pressures from a user. Conf Proc IEEE Eng Med Biol Soc. 2013; 2013:910-3. PMid:24109836.

Tyson SF, Rogerson L. Assistive walking devices in nonambulant patients undergoing rehabilitation after stroke: the effects on functional mobility, walking impairments, and patients' opinion. Arch Phys Med Rehabil. 2009; 90(3):475-9. PMid:19254614. http://dx.doi.org/10.1016/j.apmr.2008.09.563.

Valadão C, Caldeira E, Bastos-Filho TF, Frizera-Neto A, Carelli R. A New Controller for a Smart Walker Based on Human-Robot Formation. Sensors (Basel). 2016; 16(7):1116. PMid:27447634. http://dx.doi.org/10.3390/s16071116.

Verma R, Arya KN, Sharma P, Garg RK. Understanding gait control in post-stroke: implications for management. J Bodyw Mov Ther. 2012; 16(1):14-21. PMid:22196422. http://dx.doi. org/10.1016/j.jbmt.2010.12.005.

Whittle MW. Gait analysis: an introduction. 4th ed. New York: Elsevier; 2007.

World Health Organization - WHO. The top 10 causes of death, 2014 [internet]. Genève: WHO; 2015. [cited 2017 Apr 13]. Available from: http://www.who.int/mediacentre/ factsheets/fs $310 / \mathrm{en} /$ 